\title{
CORRECTIONS
}

\section{Acute coronary syndromes}

This Clinical Review contains an error (BMJ 2015;351:h5153, doi:10.1136/bmj.h5153). The fifth sentence in the section titled "Non-ST elevation myocardial infarction and unstable angina" should begin by saying: "Anticoagulation with subcutaneous fondaparinux $2.5 \mathrm{mg}$ daily ..." [not "Anticoagulation with oral fondaparinux $2.5 \mathrm{mg}$ daily ..." as was published].

Cite this as: BMJ 2015;351:h5849

๑ BMJ Publishing Group Ltd 2015 